Original Article

\title{
Clinicopathologic Characteristics and Clinical Outcome of Localized Liposarcoma: A Single-Center Experience over 25 Years and Evaluation of PD-L1 Expression
}

\author{
Heejung Chae ${ }^{1,2}$, Jeong Eun Kim', Wanlim Kim ${ }^{3}$, Jong-Seok Lee ${ }^{3}$, Si Yeol Song ${ }^{4}$, Min Hee Lee ${ }^{5}$, Hye Won Chung ${ }^{5}$, Kyung-Ja Cho ${ }^{6}$, \\ Joon Seon Song ${ }^{6}$, Jin-Hee Ahn ${ }^{1}$ \\ ${ }^{1}$ Department of Oncology, Asan Medical Center, University of Ulsan College of Medicine, Seoul, ${ }^{2}$ Center for Breast Cancer, National Cancer Center \\ Korea, Goyang, Departments of ${ }^{3}$ Orthopedic Surgery, ${ }^{4}$ Radiation Oncology, ${ }^{5}$ Radiology, and ${ }^{6}$ Pathology, Asan Medical Center, University of \\ Ulsan College of Medicine, Seoul, Korea
}

Purpose For liposarcoma (LPS), clinical course and proper treatment strategies have not been well-established. Recently, immunecheckpoint inhibitors have shown potential efficacy in LPS. We aimed to describe the clinical course of LPS and evaluate the clinical impact of programmed death-ligand 1 (PD-L1).

Materials and Methods We reviewed all consecutive patients $(n=332)$ who underwent curative-intent surgery for localized LPS at Asan Medical Center between 1989 and 2017. PD-L1 testing was performed in well-differentiated and dedifferentiated LPS.

Results The median age was 56 years with males comprising 60.8\%. Abdomen-pelvis (47.6\%) and well-differentiated (37.7\%) were the most frequent primary site and histologic subtype, respectively. During a median follow-up of 81.2 months, recurrence was observed in 135 (40.7\%), and 86.7\% (117/135) were loco-regional. Well-differentiated subtype (hazard ratio [HR], 0.38), abdomenpelvis origin (HR, 2.43), tumor size larger than $5 \mathrm{~cm}(\mathrm{HR}, 1.83)$, positive resection margin (HR, 2.58), and postoperative radiotherapy (HR, 0.36) were significantly related with recurrence-free survival as well as visceral involvement (HR, 1.84) and multifocality (HR, $3.79)$ in abdomen-pelvis LPS. PD-L1 was positive in 31.5\% (23/73) and 51.3\% (39/76) of well-differentiated and dedifferentiated LPS, respectively, but had no impact on survival outcomes.

Conclusion Clinical course of LPS was heterogeneous according to histology and anatomic location. Clear resection margin was important to lower recurrence and postoperative radiotherapy might have additional benefit. A decent portion of well-differentiated and dedifferentiated LPS were positive for PD-L1, but its prognostic role was unclear. Further research is needed to determine clinical implications of PD-L1, especially for advanced-stage LPS with unmet needs for effective systemic treatment.

Key words Sarcoma, Liposarcoma, Retrospective analysis, Prognosis, Immunotherapy, Programmed death-ligand 1

\section{Introduction}

Liposarcoma (LPS) is a mesenchymal-origin cancer arising from precursors of adipocytes and accounts for $15 \%$ to $20 \%$ of all adult malignant soft-tissue sarcoma [1]. It can arise in any fat-containing region of the body, and is histologically classified into four subtypes; well-differentiated, dedifferentiated, myxoid/round and pleomorphic LPS [2]. Due to its rarity and the heterogeneity in tumor location and histology, the clinical characteristics, and oncologic outcomes of LPS, along with an appropriate proper treatment strategy for it, have not been well-established.

The mainstay of treatment for localized LPS is still surgical resection, but tumor recurrence is common even after surgery with curative intent. Other treatment modalities, including radiation therapy and systemic chemotherapy, are used in the multidisciplinary management of LPS. Radiation therapy has been recommended in soft-tissue sarcomas with large size, high-grade (especially myxoid subtype), positive resection margins, or extremity origin for better local control. However, its survival benefit remains unclear, and there is still no full consensus on the optimal indications for adjuvant radiation therapy in other histologic subtype or abdominalpelvis LPS $[3,4]$. Conventional cytotoxic chemotherapy has different benefit based on histologic subtypes and anatomic sites of LPS. Patients with myxoid histology and extremity origin has known to be chemo-sensitive with a response rate of 40\%-50\% [5]. On the other hand, chemotherapy has limited efficacy in other histologic subtypes with a response rate of about $10 \%$ and is not generally recommended in the
Correspondence: Jin-Hee Ahn

Department of Oncology, Asan Medical Center, University of Ulsan College of Medicine, 88 Olympic-ro 43-gil, Songpa-gu, Seoul 05505, Korea

Tel: 82-2-3010-3222 Fax: 82-2-3010-6961 E-mail: drjiny@amc.seoul.kr

Received April 22, 2021 Accepted July 5, 2021 Published Online July 6, 2021
Co-correspondence: Joon Seon Song

Department of Pathology, Asan Medical Center, University of Ulsan College of Medicine, 88 Olympic-ro 43-gil, Songpa-gu, Seoul 05505, Korea Tel: 82-2-3010-4548 Fax: 82-2-472-7898 E-mail: songjs@amc.seoul.kr *Heejung Chae and Jeong Eun Kim contributed equally to this work. 
preoperative or postoperative setting [6-8]. Recently, it was suggested that immune-checkpoint inhibitors may have clinical activity for soft-tissue sarcomas in phase II trials that included patients with well-differentiated and dedifferentiated LPS $[9,10]$. However, it has not been comprehensively investigated whether the expression of programmed deathligand 1 (PD-L1), one of the well-established predictors for response to immune-checkpoint inhibitors, is common in LPS and how PD-L1 positivity is related with patient outcome.

In the present single-center retrospective study, we aimed to determine the clinical course and treatment outcome of localized LPS and evaluate the expression level of PD-L1 and determine its relationship with clinical outcomes in well-differentiated and dedifferentiated LPS.

\section{Materials and Methods}

\section{Patients}

We retrospectively reviewed all consecutive patients who were treated for LPS at Asan Medical Center, Seoul, South Korea between July 1989 and January 2018 using the clinical database system of Asan Medical Center (Asan BiomedicaL Research Environment, ABLE) after extracting all records containing phrase "liposarcoma". Patients whose diagnoses were not pathologically confirmed or whose electronic medical records were not available from initial diagnosis were excluded from the final analysis. Patients who had stage IV disease and did not receive curative-intent surgical resection were also not included. Clinical data regarding demographic factors, baseline tumor characteristics, treatment history and survival outcomes were retrospectively obtained by reviewing the anonymous records from ABLE.

Pathologic diagnosis of LPS and its histologic classification were made by expert sarcoma pathologists (K-J.C., J.S.S.) based on the World Health Organization (WHO) classification system. The treatment plan was decided on multidisciplinary approach at tertiary referral cancer in accordance with the international guidelines. Surgeons and radiologists determined the optimal extent of resection to achieve clear resection margin and to preserve the adjacent critical structures. Experienced medical oncologists and radiation oncologists determined the need of postoperative radiotherapy and chemotherapy, considering tumor location, tumor size, histologic subtype, grade, adjacent structures as well as resection margin status.

\section{PD-L1 staining}

When available, formalin fixed paraffin-embedded tumor samples collected from patients with well-differentiated and dedifferentiated LPS during a previous biopsy or surgery were reviewed by a designated pathologist and analyzed for PD-L1 expression. Immunohistochemical staining for PD-L1 was performed with the Ventana SP263 assay (rabbit monoclonal primary anti-PD-L1 antibody, Ventana Medical Systems, Tucson, AZ), Food and Drug Administration (FDA)approved complementary diagnostics [11]), on the Benchmark XT staining systems and Ultra with the OptiView Universal DAB Detection Kit (Ventana Medical Systems), according to the manufacturer's instructions. Whole tumor section was stained in each case to adequately reflect heterogeneity of expression. Positive PD-L1 expression was defined as staining in $\geq 1 \%$ of tumor cells.

\section{Statistical analysis}

Overall survival (OS) was defined as the time between the diagnosis and death from any cause. Recurrence-free survival (RFS) was defined as the duration between the resection with curative intent until tumor recurrence or death from any cause, whichever came first. When recurrence or death were not observed, survival time was censored at the date of the last follow-up visit. Survival outcomes were estimated using the Kaplan-Meier method and compared using the log-rank test. Multivariate analyses for survival outcomes were analyzed using the Cox proportional hazards model. A p-value less than 0.05 was considered statistically significant. Statistical package for the Social Sciences ver. 22.0 (IBM Corp., Armonk, NY) was used for all statistical analyses.

\section{Results}

Out of 467 patients identified from the database, 332 patients who had underwent curative-intent resection for pathologically-confirmed localized LPS and had sufficient medical records were finally included in this retrospective analysis and 135 patients were excluded for following reasons: medical records not available $(\mathrm{n}=71)$, diagnosed and treated at other institution $(\mathrm{n}=26)$, follow-up loss after diagnosis $(n=24)$, distant metastasis at diagnosis $(n=12)$, or no surgery due to high perioperative risk $(n=2)$. Baseline characteristics of all patients evaluated in this study are summarized in Table 1 . The median age of the patients was 56 years (range, 19 to 87 years) with males comprising 60.8\% (202/332) of patients. The most common subtype was well-differentiated LPS $(125 / 332,37.7 \%)$, followed by dedifferentiated (103/332, $31.0 \%)$, myxoid/round (91/332, 27.4\%), and pleomorphic $(13 / 332,3.9 \%)$. By primary site, abdomen-pelvis (158/332, $47.6 \%)$ was most frequently involved; extremity $(136 / 332$, $41.0 \%)$, thorax $(22 / 332,6.6 \%)$ and head-neck $(16 / 332,4.8 \%)$ came next. 
Table 1. Baseline characteristics and treatment summary after surgery of patients with localized disease at diagnosis

\begin{tabular}{|c|c|c|c|c|c|}
\hline Characteristic & $\begin{array}{c}\text { Total } \\
(n=332)\end{array}$ & $\begin{array}{l}\text { Head-neck } \\
\qquad(n=16)\end{array}$ & $\begin{array}{l}\text { Thorax } \\
(n=22)\end{array}$ & $\begin{array}{l}\text { Abdomen-pelvis } \\
\qquad(\mathrm{n}=158)\end{array}$ & $\begin{array}{l}\text { Extremity } \\
(n=136)\end{array}$ \\
\hline Age (yr) & $56(19-87)$ & $58(34-84)$ & $51(19-73)$ & $58(27-87)$ & $54(20-81)$ \\
\hline \multicolumn{6}{|l|}{ Sex } \\
\hline Male & $202(60.8)$ & $13(81.3)$ & $15(68.2)$ & $94(59.5)$ & $80(58.8)$ \\
\hline Female & $130(39.2)$ & $3(18.8)$ & $7(31.8)$ & $64(40.5)$ & $56(41.2)$ \\
\hline \multicolumn{6}{|l|}{ Histologic subtype } \\
\hline Well-differentiated & $125(37.7)$ & $11(68.8)$ & $4(18.2)$ & $50(31.6)$ & $60(44.1)$ \\
\hline Dedifferentiated & $103(31.0)$ & $1(6.3)$ & $5(22.7)$ & $83(52.5)$ & $14(10.3)$ \\
\hline Myxoid/Round & $91(27.4)$ & $4(25.0)$ & $10(45.5)$ & $19(12.0)$ & $58(42.6)$ \\
\hline Pleomorphic & $13(3.9)$ & 0 & 3 (13.6) & $6(3.8)$ & $4(2.9)$ \\
\hline \multicolumn{6}{|l|}{ Tumor size $(\mathrm{cm})$} \\
\hline$\leq 5$ & $54(16.3)$ & $4(25.0)$ & $5(22.7)$ & $25(15.8)$ & $20(14.7)$ \\
\hline$>5$ and $\leq 10$ & $90(27.1)$ & $7(43.8)$ & $5(22.7)$ & $33(20.9)$ & $45(33.1)$ \\
\hline$>10$ and $\leq 15$ & $80(24.1)$ & $2(12.5)$ & $6(27.3)$ & $43(27.2)$ & $29(21.3)$ \\
\hline$>15$ & $108(32.5)$ & $3(18.8)$ & $6(27.3)$ & $57(36.1)$ & $42(30.9)$ \\
\hline \multicolumn{6}{|l|}{ Resection margin } \\
\hline R0 & $190(57.2)$ & $8(50.0)$ & $16(72.7)$ & $64(40.5)$ & $102(75.0)$ \\
\hline $\mathrm{R} 1$ & $99(29.8)$ & $6(37.5)$ & $5(22.7)$ & $62(39.2)$ & $26(19.1)$ \\
\hline $\mathrm{R} 2$ & $19(5.7)$ & 0 & 0 & $17(10.8)$ & $2(1.5)$ \\
\hline Indeterminate & $24(7.2)$ & $2(12.5)$ & $1(4.5)$ & $15(9.5)$ & $6(4.4)$ \\
\hline \multicolumn{6}{|l|}{ Postoperative treatment } \\
\hline Radiotherapy & $111(33.4)$ & $7(43.8)$ & $10(45.5)$ & $49(31.0)$ & $45(33.1)$ \\
\hline Chemotherapy & $17(5.1)$ & 0 & $1(4.5)$ & $15(9.5)$ & $1(0.7)$ \\
\hline Radiotherapy and chemotherapy & $29(8.7)$ & 0 & $1(4.5)$ & $5(3.2)$ & $23(16.9)$ \\
\hline None & $175(52.7)$ & $9(56.3)$ & $10(45.5)$ & $89(56.3)$ & $67(49.3)$ \\
\hline \multicolumn{6}{|l|}{ Recurrence } \\
\hline Yes & $135(40.7)$ & $5(31.3)$ & $11(50.0)$ & $98(62.0)$ & $21(15.4)$ \\
\hline Local & $117 / 135$ (86.7) & $5 / 5(100)$ & $8 / 11(72.7)$ & $89 / 98$ (90.8) & $15 / 21(71.4)$ \\
\hline Distant & $18 / 135(13.3)$ & 0 & $3 / 11(27.3)$ & 9/98 (9.2) & 6/21 (28.6) \\
\hline No & $197(59.3)$ & $11(68.8)$ & $11(50.0)$ & $60(38.0)$ & 115 (84.6) \\
\hline
\end{tabular}

Values are presented as median (range) or number $(\%)$.

\section{Treatment and clinical outcomes}

All included patients underwent curative-intent surgery as the first treatment for their localized LPS; no one received preoperative chemotherapy or radiotherapy. Information on treatment and recurrence is summarized in Table 1 . The R0 resection rate was 57.2\% (190/332), and about half (157/332, $47.3 \%$ ) of patients underwent postoperative treatment consisting of radiotherapy $(111 / 332,33.4 \%)$, chemotherapy $(17 / 332,5.1 \%)$, or both $(29 / 332,8.7 \%)$. During a median follow-up duration of 81.2 months, recurrence was observed in 135 patients $(40.7 \%)$ after surgery, and $86.7 \%$ (117/135) of recurrences were local. Recurrence pattern was slightly different between histologic subtypes. Dedifferentiated and pleomorphic LPS had relatively high recurrence rate $(63.1 \%$ and $61.5 \%$ ) compared with well-differentiated $(30.4 \%)$ and myxoid/round (26.4\%) LPS, but distant recurrence was con- sistently observed in less than $10 \%$ across all subtypes (S1 Table).

Median OS and RFS of the whole cohort were 198.4 (95\% CI, not available) and 82.9 months (range, 46.9 to 118.8 months), respectively. Age $>60$ years was significantly associated with poorer survival outcomes (vs. $\leq 60$ years; median RFS, 48.6 vs. 130.9 months, $\mathrm{p}=0.001$; median OS, 198.4 vs. not reached, $\mathrm{p}=0.009$ ) (Fig. 1A and B). By histology, well-differentiated LPS (median RFS, 124.6 months; median OS, 198.4 months) and myxoid/round LPS (median RFS and OS, not reached) tended to have better prognosis than dedifferentiated LPS (median RFS, 23.4 months; median OS, 82.8 months) and pleomorphic LPS (median RFS, 26.6 months; median OS, 33.9 months) (Fig. 1C and D). LPS originating in the abdomen-pelvis (median RFS, 29.4 months; median OS, 126.2 months) had the poorest survival, while LPS originating 
A
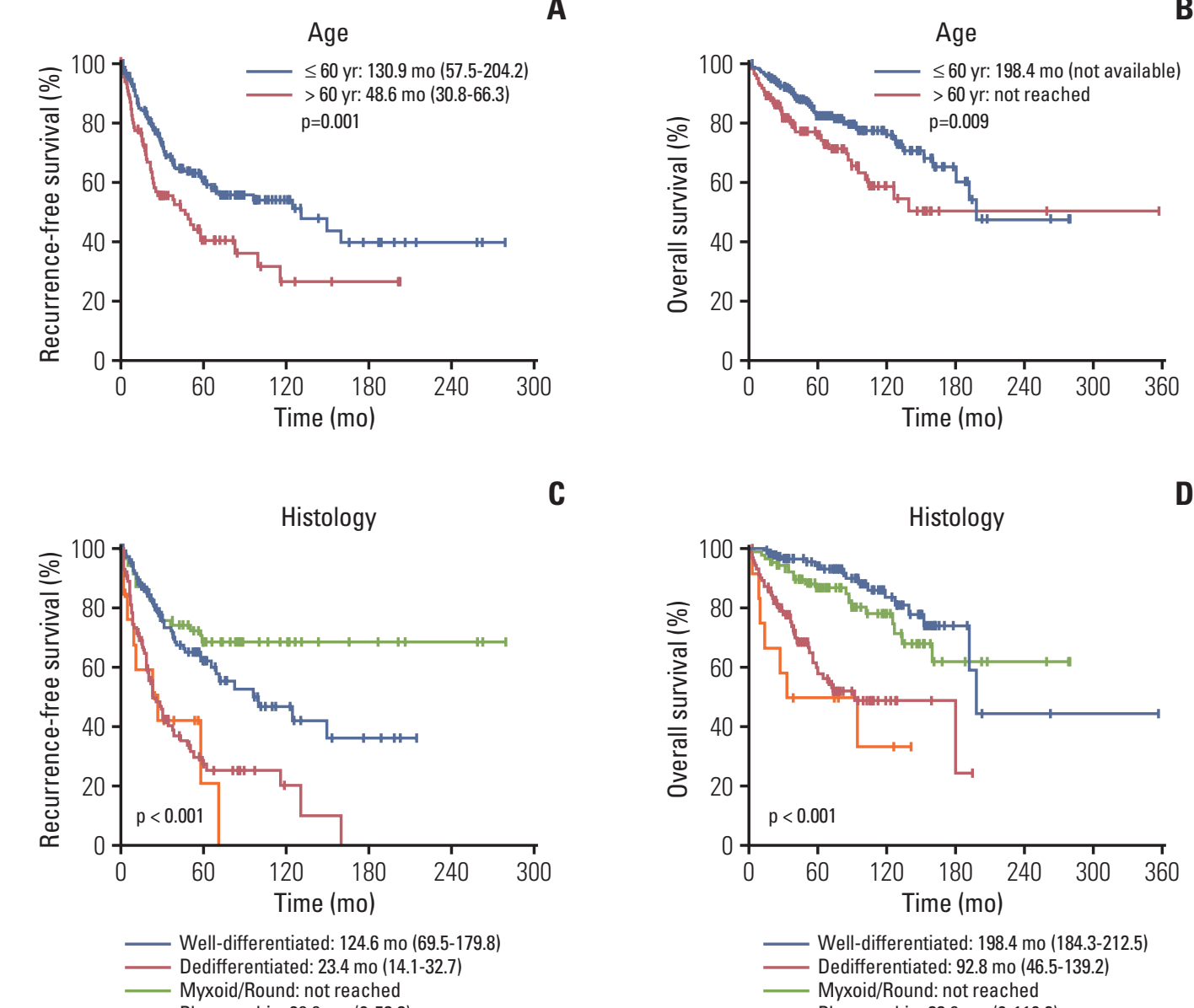

C

D

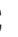

Myxoid/Round: not reached

Pleomorphic: 26.6 mo (0-53.3)

$\mathbf{E}$
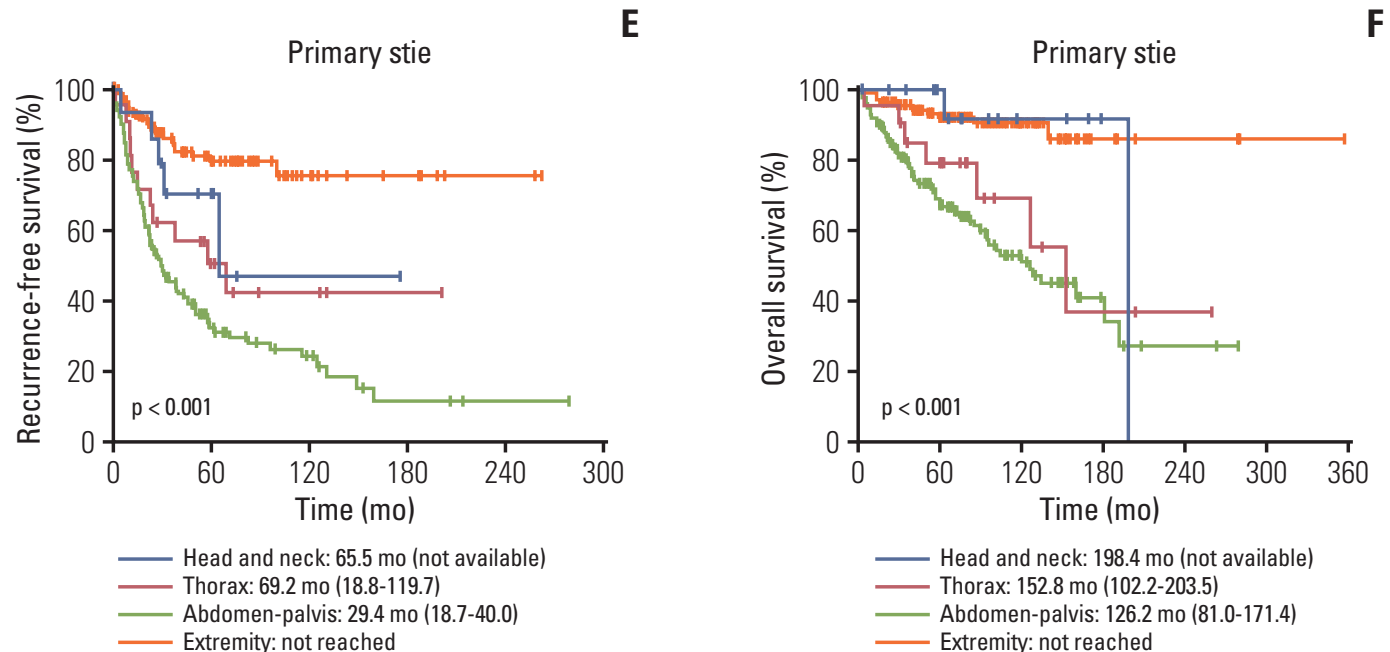

Fig. 1. Survival outcomes of localized liposarcoma (LPS) after surgery according to cancer characteristics. Recurrence-free survival and overall survival curve of patients with localized LPS according to age (A, B), histologic subtype (C, D), primary tumor site (E, F), and primary tumor size $(\mathrm{G}, \mathrm{H})$, the estimated median survival time $(95 \%$ confidence interval) is presented underneath the graphs. (Continued to the next page) 

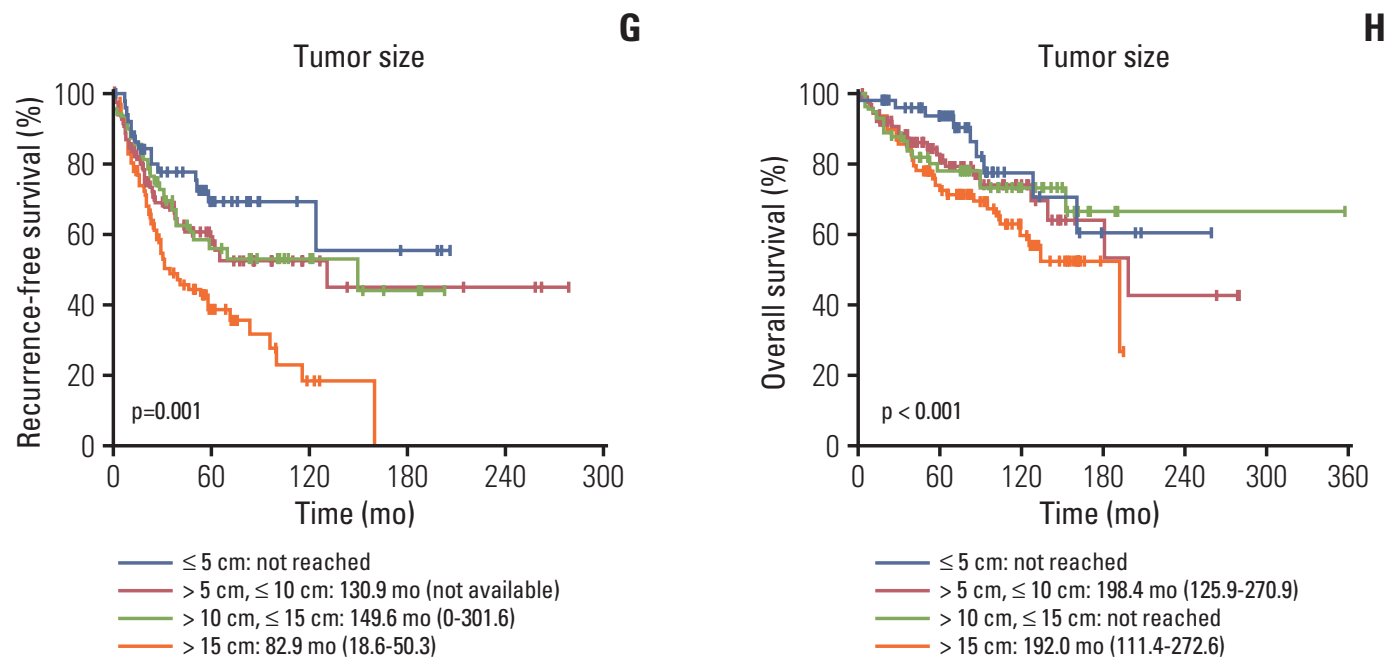

Fig. 1. (Continued from the previous page)

in the extremity had most favorable survival (median RFS and OS, not reached) (Fig. 1E and F). Larger tumor was also associated with shorter RFS ( $\leq 5 \mathrm{~cm}$ vs. $>15 \mathrm{~cm}$, not reached vs. 82.9 months, $\mathrm{p}=0.001$ ) and $\mathrm{OS}$ (not reached vs. 192.0 months, $\mathrm{p}<0.001$ ) (Fig. 1G and H). Regarding treatment, resection margin status was key factor determining RFS (R0 vs. R1 vs. R2, not reached vs. 40.3 months vs. 9.3 months, $\mathrm{p}<$ 0.001) and OS (R0 vs. R1 vs. R2, not reached vs. 198.4 months vs. 39.5 months, $\mathrm{p}<0.001$ ) (Fig. $2 \mathrm{~A}$ and B). Furthermore, adjuvant radiotherapy resulted in longer RFS (vs. no adjuvant radiotherapy; 149.6 months vs. 37.3 months, $\mathrm{p}<0.001$ ) but had less impact on extending OS (198.4 months vs. 192.0 months, $\mathrm{p}=0.043$ ) (Fig. 2C and D). On the other hand, adjuvant chemotherapy tended to improve RFS (vs. no adjuvant chemotherapy; 159.9 months vs. 65.5 months, $\mathrm{p}=0.282$ ) and OS (not reached vs. 192.0 months, $\mathrm{p}=0.368$ ), but these differences were not statistically significance (Fig. 2E and F). In multivariate analysis, histology, primary site, resection margin status and postoperative radiotherapy remained significantly associated with RFS and OS, while age only remained significantly associated with OS (Table 2). Tumor size larger than $5 \mathrm{~cm}$ was associated with shorter RFS (hazard ratio [HR], 1.83) with marginal p-value of 0.046 , but it was not led to the shortening of OS (not retained in multivariate analysis). When analyzed by primary site, positive microscopic resection margin status (R1 resection) was associated with shorter RFS and OS compared with clear resection margin (R0 resection) in abdomen-pelvis LPS, while it did not affect both RFS and OS in extremity LPS (S2 Fig.). Postoperative radiotherapy extended RFS and OS abdomen-pelvis LPS. In extremity LPS, it extended RFS, but longer RFS did not lead to better OS (S3 Fig.).

\section{Abdominal-pelvis LPS}

Among patients with localized LPS originating in the abdomen-pelvis $(\mathrm{n}=158)$, visceral involvement was observed in $31.0 \%$ (49/158), and $12.0 \%$ of patients $(19 / 158)$ had multifocal disease. Visceral involvement, which was defined as microscopic or macroscopic tumor invasion into the adjacent organs such as kidney, colon, pancreas, liver, bladder, and adrenal grand, was associated with shorter RFS (vs. no visceral involvement, 18.2 months vs. 46.7 months, $\mathrm{p}<0.001$ ) and OS (56.3 months vs. 181.0 months, $\mathrm{p}<0.001$ ) (S4A and S4B Fig.). Compared with patients having a solitary abdomin-pelvic lesion, patients with multifocal disease had significantly poorer RFS (8.7 months vs. 34.4 months, $\mathrm{p}<$ 0.001 ) and $O S$ ( 37.7 months vs. 128.3 months, $\mathrm{p}=0.002$ ) (S4C and S4D Fig.). The negative prognostic impact of visceral involvement and multifocality remained significant in a multivariate analysis with age, histology, resection margin, and postoperative radiotherapy (S5 Table).

\section{PD-L1 expression in localized LPS}

A total of 149 patients who were pathologically diagnosed with well-differentiated $(n=73)$ or dedifferentiated $(n=76)$ LPS and had tissue available for immunohistochemical staining were included in this analysis for PD-L1 expression (Fig. $3 \mathrm{~A}-\mathrm{C}$ ). The PD-L1 positive (tumor proportion score $\geq 1 \%$ ) rate was $31.5 \%$ and $51.3 \%$ in well-differentiated and dedifferentiated LPS, respectively. There were no significant differences in baseline characteristics or treatment between the PD-L1 positive and negative group both in well-differentiated and dedifferentiated LPS (S6 Table).

Overall, PD-L1 expression did not have an impact on RFS (vs. no expression, median 34.4 months [95\% confidence 


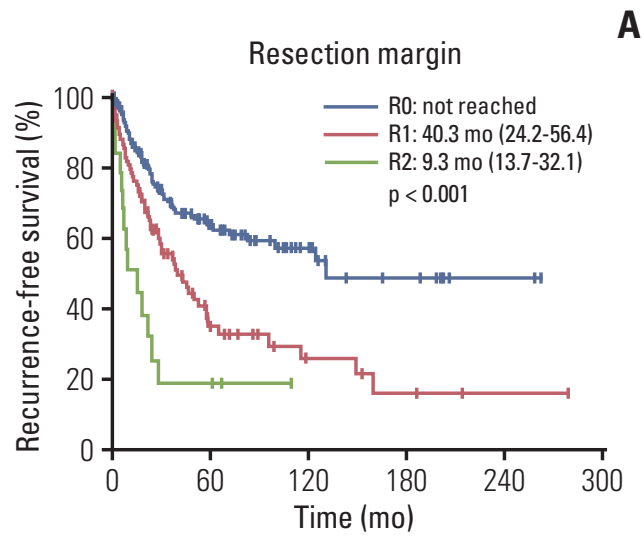

A
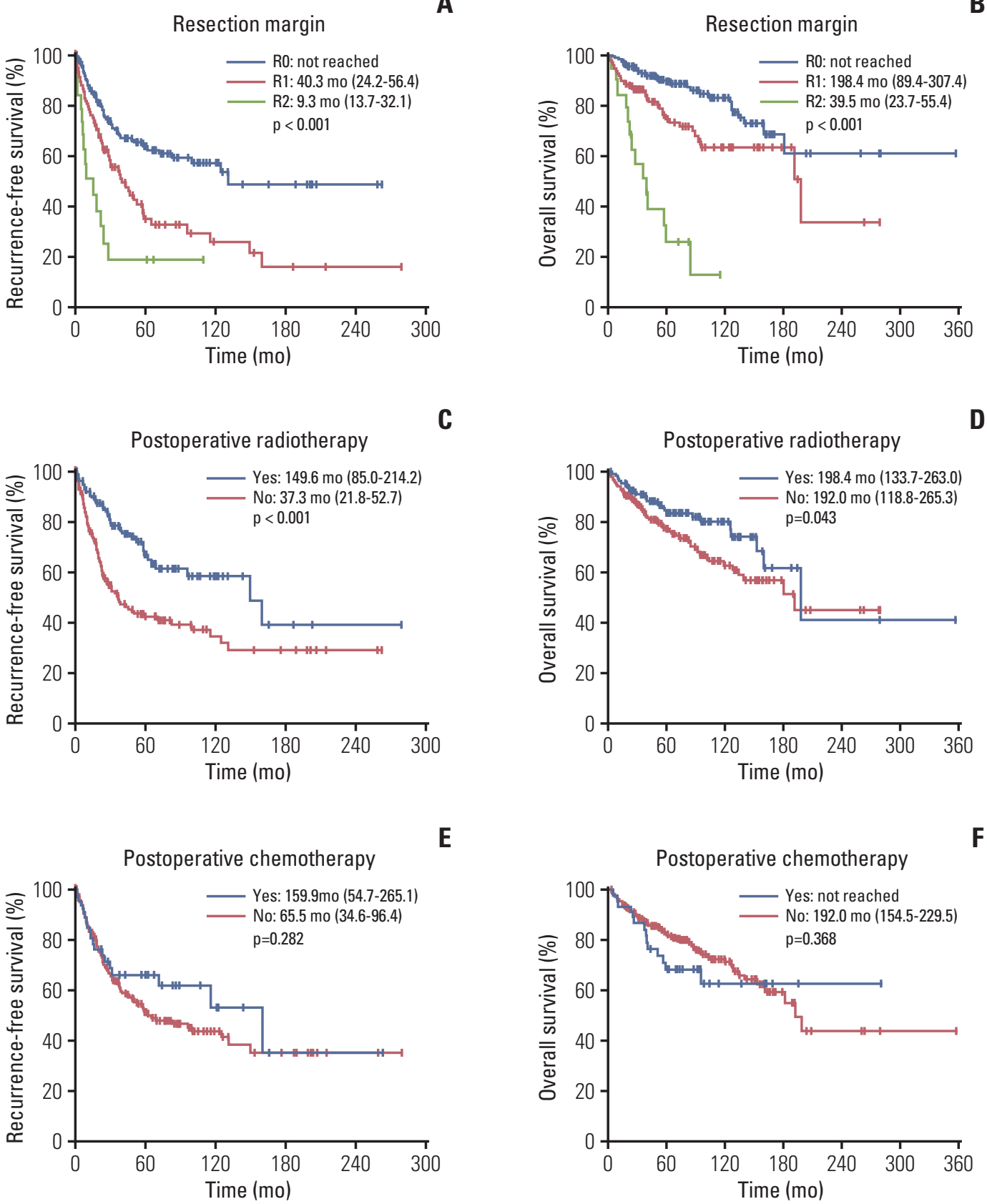

Fig. 2. Survival outcomes of localized liposarcoma (LPS) after surgery according to applied treatment. Recurrence-free survival and overall survival curve of patients with localized LPS according to resection margin (A, B), postoperative radiotherapy (C, D), and postoperative chemotherapy $(\mathrm{E}, \mathrm{F})$, the estimated median survival time ( $95 \%$ confidence interval) is presented underneath the graphs.

interval (CI), 24.0 to 44.9$]$ vs. 37.4 months [95\% CI, 16.5 to 58.2$]$, $\mathrm{p}=0.602$ ) and $\mathrm{OS}$ (vs. no expression, median not reached vs. 181.0 months [ $95 \% \mathrm{CI}, 72.4$ to 289.6], $\mathrm{p}=0.539$ ) (Fig. 3D and E). A subgroup analysis was additionally performed by histologic type (S7 Fig.). In well-differentiated LPS, PD-L1 expression was associated with shorter RFS (vs. no expression, 31.3 months [95\% CI, 23.5 to 39.2] vs. 99.8 months [95\% CI, 63.4 to 136.2], $\mathrm{p}=0.023$ ), which remained significant in the multivariate analysis (HR, 2.68 [95\% CI, 1.10 to 6.55], $\mathrm{p}=0.030$ ). In dedifferentiated LPS on the other hand, PD-L1 positive patients had longer RFS (vs. PD-L1 negative patients, 34.3 months [ $95 \%$ CI, 21.9 to 46.9 ] vs. 18.3 months [95\% CI, 15.7 
Table 2. Multivariate analysis for survival outcomes in localized LPS

\begin{tabular}{|c|c|c|c|c|}
\hline \multirow{2}{*}{ Variable } & \multicolumn{2}{|c|}{ RFS } & \multicolumn{2}{|c|}{ OS } \\
\hline & $\mathrm{HR}(95 \% \mathrm{CI})$ & p-value & HR $(95 \%$ CI $)$ & p-value \\
\hline Age $>60$ yr & \multicolumn{2}{|c|}{ Not retained } & $1.64(1.02-2.64)$ & 0.043 \\
\hline Histology, well-differentiated & $0.38(0.25-0.59)$ & $<0.001$ & $0.30(0.17-0.54)$ & $<0.001$ \\
\hline Primary site, abdomen-pelvis & $2.43(1.58-3.71)$ & $<0.001$ & $2.42(1.37-4.28)$ & 0.002 \\
\hline Size $>5 \mathrm{~cm}$ & $1.83(1.01-3.31)$ & 0.046 & \multicolumn{2}{|c|}{ Not retained } \\
\hline Resection margin, R1/2 & $2.58(1.75-3.80)$ & $<0.001$ & $2.29(1.37-3.83)$ & 0.002 \\
\hline Postoperative radiotherapy & $0.36(0.24-0.53)$ & $<0.001$ & $0.57(0.34-0.94)$ & 0.029 \\
\hline
\end{tabular}

CI, confidence interval; HR, hazard ratio; LPS, liposarcoma; OS, overall survival; RFS, recurrence-free survival.

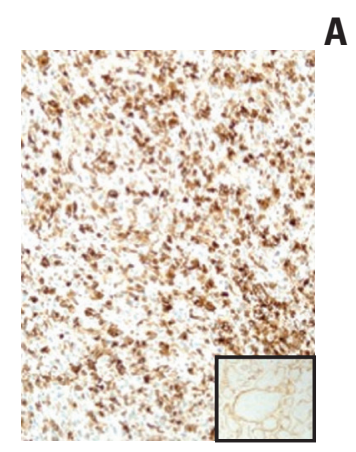

A

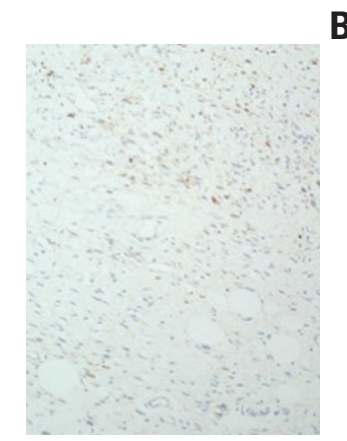

B

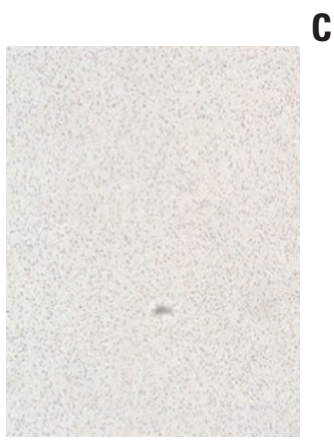

D
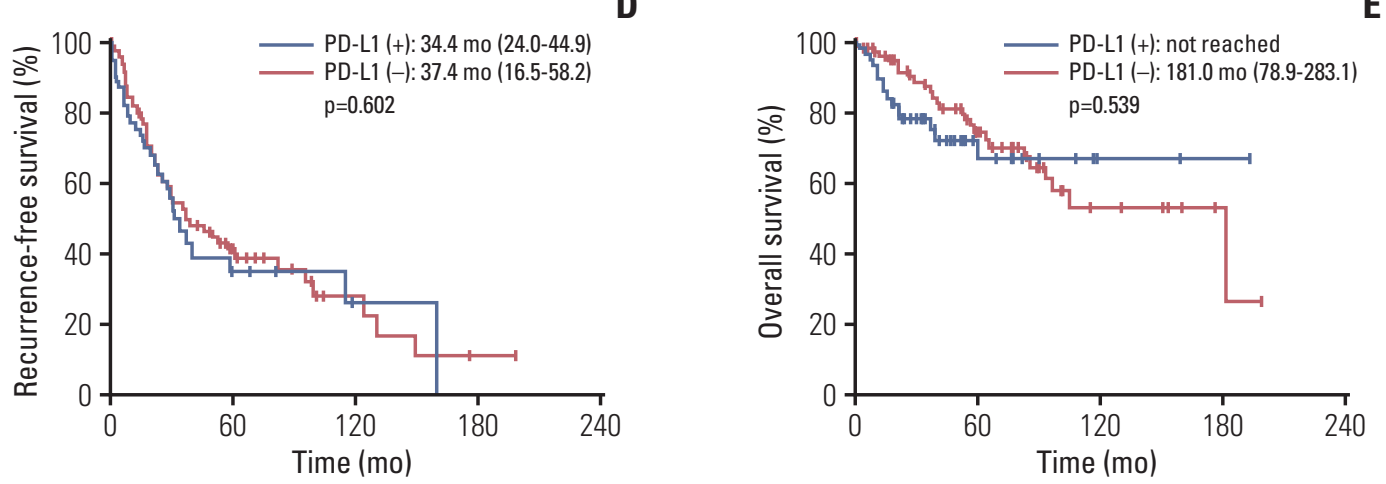

Fig. 3. Representative images of programmed death-ligand 1 (PD-L1) expression and survival outcomes according to PD-L1 status. (A) Dedifferentiated liposarcoma (LPS) shows immunopositivity for PD-L1 (SP263) with diffuse and homogenous pattern $(\times 200)$. Placenta tissue is used as positive control (inset). (B) Well-differentiated LPS shows patchy immunoreactivity for PD-L1 (SP263) ( $\times 200)$. (C) Tumor cells of dedifferentiated LPS are negative for PD-L1 (SP263) (×100). There was no difference in recurrence-free survival (D) and overall survival curve (E) according to PD-L1 status. The estimated median survival time (95\% confidence interval) is presented underneath the graphs.

to 21.0], $\mathrm{p}=0.032)$, which showed statistical significance $(H R$, 0.46 [95\% CI, 0.24 to 0.87]; $\mathrm{p}=0.017$ ) in the multivariate analysis (Table 3). OS was not significantly different according to PD-L1 expression both in well-differentiated and dedifferentiated LPS.

\section{Discussion}

This single-center retrospective study described the longterm clinical courses of 332 localized LPS patients after surgery and evaluated prognostic factors for survival outcomes after curative-intent surgery. The most common histologic subtype and primary tumor site in this cohort were welldifferentiated LPS (37.7\%) and the abdomen-pelvis (47.6\%), respectively. We found that age, histologic subtype, primary 
Table 3. Multivariate analysis including PD-L1 status for RFS

\begin{tabular}{|c|c|c|c|c|c|c|c|c|}
\hline \multirow{3}{*}{ Variable } & \multicolumn{4}{|c|}{ Well-differentiated LPS } & \multicolumn{4}{|c|}{ Dedifferentiated LPS } \\
\hline & \multicolumn{2}{|c|}{ RFS } & \multicolumn{2}{|l|}{ OS } & \multicolumn{2}{|l|}{ RFS } & \multicolumn{2}{|l|}{ OS } \\
\hline & HR $(95 \% \mathrm{CI})$ & p-value & HR $(95 \% \mathrm{CI})$ & p-value & HR $(95 \%$ CI $)$ & p-value & HR $(95 \% \mathrm{CI})$ & p-value \\
\hline Age $>60 \mathrm{yr}$ & \multicolumn{2}{|c|}{ Not retained } & $3.34(0.73-13.47)$ & 0.090 & $2.08(1.11-3.90)$ & 0.022 & $2.24(1.02-4.93)$ & 0.045 \\
\hline $\begin{array}{l}\text { Primary site, } \\
\text { abdomen-pelvis }\end{array}$ & \multicolumn{2}{|c|}{ Not retained } & $3.74(0.77-18.13)$ & 0.102 & \multicolumn{2}{|c|}{ Not retained } & \multicolumn{2}{|c|}{ Not retained } \\
\hline Size $>5 \mathrm{~cm}$ & \multicolumn{2}{|c|}{ Not retained } & \multicolumn{2}{|c|}{ Not retained } & $8.08(1.84-35.43)$ & 0.006 & $3.18(0.73-13.93)$ & 0.124 \\
\hline Resection margin, R1/2 & $2.93(1.31-6.55)$ & 0.009 & \multicolumn{2}{|c|}{ Not retained } & $2.35(1.21-4.56)$ & 0.012 & 3.57 (1.39-9.19) & 0.008 \\
\hline $\begin{array}{l}\text { Postoperative } \\
\text { radiotherapy }\end{array}$ & \multicolumn{2}{|c|}{ Not retained } & \multicolumn{2}{|c|}{ Not retained } & $0.39(0.21-0.73)$ & 0.004 & $0.41(0.18-0.93)$ & 0.032 \\
\hline PD-L1, positive & $2.68(1.10-6.55)$ & 0.030 & \multicolumn{2}{|c|}{ Not retained } & $0.46(0.24-0.87)$ & 0.017 & \multicolumn{2}{|c|}{ Not retained } \\
\hline
\end{tabular}

CI, confidence interval; HR, hazard ratio; LPS, liposarcoma; OS, overall survival; PD-L1, programmed death-ligand 1; RFS, recurrence-free survival.

Table 4. Previous reports on PD-L1 expression rates in sarcoma patients

\begin{tabular}{|c|c|c|c|c|c|}
\hline Author, year & $\begin{array}{l}\text { Overall } \\
\text { patients }\end{array}$ & $\begin{array}{c}\text { LPS } \\
\text { patients }\end{array}$ & $\begin{array}{c}\text { PD-L1 } \\
\text { positivity }(\%)\end{array}$ & PD-L1 antibody & $\begin{array}{l}\text { Cutoff for } \\
\text { positivity }\end{array}$ \\
\hline $\begin{array}{l}\text { Kim et al. } \\
(2013)[19]\end{array}$ & 105 & $\begin{array}{l}\text { Well-differentiated } 4 \\
\text { Dedifferentiated } 3 \\
\text { Myxoid } 10\end{array}$ & $\begin{array}{l}50 \\
67 \\
30\end{array}$ & $\begin{array}{l}\text { Clone H-130, Santa Cruz } \\
\text { Biotechnology, USA }\end{array}$ & Any $\%$ \\
\hline $\begin{array}{l}\text { D' Angelo et al. } \\
\text { (2015) [18] }\end{array}$ & 50 & $\begin{array}{l}\text { Well-differentiated } 1 \\
\text { Dedifferentiated } 2 \\
\text { Myxoid } 2\end{array}$ & $\begin{array}{l}0 \\
0 \\
0\end{array}$ & DAKO, USA & $>1 \%$ \\
\hline $\begin{array}{l}\text { Paydas et al. } \\
\text { (2016) [20] }\end{array}$ & 65 & $\begin{array}{l}\text { Dedifferentiated } 4 \\
\text { Myxoid } 1 \\
\text { Unspecified } 1\end{array}$ & $\begin{array}{r}25 \\
0 \\
0\end{array}$ & $\begin{array}{l}\text { CD274/ PDL1 AM26531AF-N, } \\
\text { Acris, Germany }\end{array}$ & Any $\%$ \\
\hline $\begin{array}{l}\text { Kim et al. } \\
\text { (2016) [17] }\end{array}$ & 82 & 0 & & $\begin{array}{l}\text { Clone 130021, } \\
\text { R\&D Systems, USA }\end{array}$ & $\begin{array}{l}\text { Intensity } \mathrm{x} \\
\text { proportion }>1\end{array}$ \\
\hline $\begin{array}{l}\text { Torabi et al. } \\
(2017)[24]\end{array}$ & 196 & $\begin{array}{l}\text { Well-differentiated } 23 \\
\text { Myxoid / Round } 35 \\
\text { Pleomorphic } 6\end{array}$ & $\begin{array}{c}0 \\
0 \\
16.7\end{array}$ & $\begin{array}{l}\text { Clone ab58810 and ab205921, } \\
\text { Abcam, USA; Clone E1L3N, } \\
\text { Cell Signaling Technology, USA }\end{array}$ & Any $\%$ \\
\hline $\begin{array}{l}\text { Yan et al. } \\
(2019)[22]\end{array}$ & 56 & $\begin{array}{l}\text { Well-differentiated } 16 \\
\text { Dedifferentiated } 26 \\
\text { Myxoid } 9 \\
\text { Pleomorphic } 5\end{array}$ & $\begin{array}{l}12.5 \\
30.8 \\
22.2 \\
20.0\end{array}$ & $\begin{array}{l}\text { TA809809, Beijing Zhongshan Golden } \\
\text { Bridge Biotechnology Co., China }\end{array}$ & Any $\%$ \\
\hline $\begin{array}{l}\text { Orth et al. } \\
(2020)[21]\end{array}$ & 225 & Dedifferentiated 49 & 12.2 & $\begin{array}{l}\text { Clone E1L3N, Cell Signaling } \\
\text { Technology, USA }\end{array}$ & $>1 \%$ \\
\hline
\end{tabular}

LPS, liposarcoma; PD-L1, programmed death-ligand 1.

tumor site, resection margin status, and postoperative radiotherapy independently influenced the postoperative prognosis of LPS.

OS was well stratified by the histologic subtypes. OS for patients with well-differentiated were significantly longer compared with those with dedifferentiated and pleomorphic tumors and it was a significant independent factor affection in the multivariate analysis (HR for RFS $0.45, p<0.001$; HR for OS $0.30, \mathrm{p}<0.001)$. Our findings are consistent with prior reports that well-differentiated LPS have 5-year survival rates higher than $90 \%$, while 5-year survival rates of pleomorphic or dedifferentiated variants ranges from 30\%-70\% [12].

Primary tumor site also had a significant impact on RFS and OS after adjusting for other prognostic factors in multivariate analysis. Patients with abdominal-pelvic LPS had the 
worst prognosis with a median RFS of 29.4 months and OS of 126.2 months, while those with extremity LPS showed the longest survivals (both RFS and OS not reached). Our results are in line with a prior large prospective cohort study of 801 patients which reported that primary tumor site is an independent predictor for 12-year disease specific survival in LPS (retroperitoneal vs. extremity LPS, $32 \%-53 \%$ vs. $82 \%-87 \%$, $\left.\chi^{2}=19.01, p=0.0008\right)$ [13]. Although a recent single-center retrospective study in Germany stated that primary site was not associated with prognosis, this study may be limited because it included a relatively small number of patients $(n=130)$, and extremity LPS accounted for more than $70 \%$ of study population [14]. In retroperitoneal LPS, contiguous organ involvement has been reported to be common $(26 \%-48 \%)$ and to be associated with increased risk of recurrence $[13,15]$. In our study, visceral involvement was found in about a third of abdomen-pelvis LPS patients and was an additional independent prognostic factor (RFS: HR, 1.8; $\mathrm{p}=0.014$; OS: HR, 2.40; $\mathrm{p}=0.005)$. Meanwhile, researchers from MD Anderson Cancer Center reported that multifocality was associated with worse OS in patients with retroperitoneal soft-tissue sarcoma of multiple histologies (5-year survival rate, $31 \%$ vs. $60 \% ; \mathrm{p}<0.001$ ) [16]. Here, we showed similar findings on the prognostic role of multifocality in LPS (RFS: HR, 3.97; $\mathrm{p}<$ 0.001; OS: HR, 2.44; $\mathrm{p}=0.025$ ).

Regarding treatment, complete resection with clean microscopic margins is the utmost goal of treatment and the most important factor in predicting postoperative survival. Our finding that patients with positive resection margins had much worse outcomes (RFS: HR, 2.50; $\mathrm{p}<0.001$; OS: HR, 2.29; $\mathrm{p}=0.002$ ) are concordant with such previous findings. It should be noted that clean microscopic margin status (R0 resection) prolonged both RFS and OS compared to microscopic tumor positive margin status (R1 resection) in abdomen-pelvis LPS, but microscopic margin status had no influence on RFS and OS in patients with extremity LPS. Unlike the importance of surgical resection, the benefits of using postoperative chemotherapy and radiation therapy remains controversial. In our study, adjuvant radiotherapy was associated with longer RFS (HR, 0.37; $<<0.001)$ and OS (HR, 0.57; $\mathrm{p}=0.029)$, while adjuvant chemotherapy showed no benefit to both RFS and OS. From the subgroup analysis, postoperative radiotherapy was associated with a longer RFS but not OS in extremity LPS, while patients with abdomen-pelvis LPS who received with postoperative radiotherapy had longer OS as well as RFS. One possible explanation is that lowering recurrence is more crucial for patients with abdomen-pelvis LPS because they are harder to treat with re-excision or radiotherapy due to the deep location of the tumor and its proximity to the vital organs.

Notably, our data suggest that PD-L1 is expressed in decent proportion of well-differentiated and dedifferentiated LPS, but its role as a prognostic biomarker is unclear, with conflicting results between well-differentiated and dedifferentiated histologic subtypes. It has been previously reported that PD-L1 positivity rate varies according to the histologic subtypes of sarcoma [17-24], and PD-L1 positive sarcomas tend to have poorer survival outcomes [17,19,2124], as in other solid cancers. However, those studies were limited by much heterogeneity in histologic subtypes, and a small number of patients with LPS were included (Table 4). In the present study, we evaluated the PD-L1 status with FDA-approved SP263 assay in a larger population (73 with well-differentiated LPS and 78 with dedifferentiated LPS). Although optimal cutoff value for PD-L1 expression remains unclear, we chose $>1 \%$ as like in previous sarcoma studies listed in Table 4. Thirty-one point five percentage of welldifferentiated and $51.3 \%$ of dedifferentiated LPS patients were positive for PD-L1 expression, which is rather higher than former reports. The impact of PD-L1 positivity on prognosis was inconsistent between histologic subgroups in our study. Some researchers studied tumor-infiltrating lymphocytes, programmed cell death protein 1/PD-L1 expression, and tertiary lymphoid structures in LPS tissue, and showed that immune microenvironment of LPS is heterogeneous according to histologic subtypes, tumor grade, tumor size, multifocality, and primary or recurrent status [22,25]. The studies suggested Immunotherapy might have the potential for efficacy in a subset of LPS and there have been phase II trials (SARC028 and ALLIANCE) showing the potential activity of anti-PD-L1 therapies in soft-tissue sarcoma patients $[9,10]$, and several clinical trials of immune-oncology agents and biomarker studies are ongoing [26,27]. Further studies are needed to evaluate the clinical implications of PD-L1 status as well as other biomarkers including presence of tertiary lymphoid structures and distribution of tumorinfiltrating lymphocytes in LPS. Those studies are expected to play an important role in solving the unmet needs in immunotherapeutic strategies for advanced LPS.

This study is limited by its retrospective nature, but we tried to minimize selection bias and demonstrate real-world clinical data by including all patients diagnosed with LPS. Also, although changes in the multidisciplinary management of LPS and pathologic diagnosis may have occurred over the study period, we inevitably had to include less recent patients due to the rarity of LPS. However, those limitations may be mitigated by the fact that in this study, the pathologic diagnosis of LPS and its histologic classification were confirmed by a dedicated sarcoma pathologist and treatment decisions were made in relatively good accordance with guidelines by a multidisciplinary team at an academic tertiary center. 
In conclusion, our result showed that the clinical course of LPS is heterogeneous according to its histologic subtype and primary tumor site. We also suggest that $\mathrm{R} 0$ resection is important to lower recurrence rates and adjuvant radiation therapy may provide additional benefit following surgical resection in patients with localized LPS. Also, a decent portion of well-differentiated and dedifferentiated liposarcomas were positive for PD-L1 expression, but its prognostic impact was not clear. Further research in a larger population is needed to determine the clinical implications of PD-L1 expression and the role of immune-checkpoint inhibitors in advancedstage LPS, which has limited therapeutic options.

\section{Electronic Supplementary Material}

Supplementary materials are available at Cancer Research and Treatment website (https:// www.e-crt.org).

\section{Ethical Statement}

This study was approved by the Institutional Review Board (IRB No. 2018-0751) of Asan Medical Center and performed in accordance with the ethical standards of the institutional research committee and the latest Helsinki declaration. Informed consent for the study was waived due to the retrospective nature of the analysis.

\section{Author Contributions}

Conceived and designed the analysis: Kim JE, Ahn JH.

Collected the data: Chae H, Kim JE, Ahn JH.

Contributed data or analysis tools: Kim W, Lee JS, Song SY, Lee

$\mathrm{MH}$, Chung HW, Cho KJ, Song JS.

Performed the analysis: Chae H, Kim JE.

Wrote the paper: Chae H, Kim JE.

Edited and reviewed the manuscript: Song JS, Ahn JH.

\section{Conflicts of Interest}

Conflict of interest relevant to this article was not reported.

\section{Acknowledgments}

This study was supported by a grant from Asan Institute for Life Sciences, Asan Medical Center, Seoul, Korea (\#2018-0570) and a grant 'Elimination of Cancer Project Fund' from Asan Cancer Institute of Asan Medical Center, Seoul, Korea. The funding source had no role in study design, collection and analysis of data, or writing of the report.

\section{References}

1. Ducimetiere F, Lurkin A, Ranchere-Vince D, Decouvelaere $\mathrm{AV}$, Peoc'h M, Istier L, et al. Incidence of sarcoma histotypes and molecular subtypes in a prospective epidemiological study with central pathology review and molecular testing. PLoS One. 2011;6:e20294.

2. Jo VY, Fletcher CD. WHO classification of soft tissue tumours: an update based on the 2013 (4th) edition. Pathology. 2014;46:95-104.

3. Kaushal A, Citrin D. The role of radiation therapy in the management of sarcomas. Surg Clin North Am. 2008;88:629-46.

4. Chung PW, Deheshi BM, Ferguson PC, Wunder JS, Griffin $\mathrm{AM}$, Catton $\mathrm{CN}$, et al. Radiosensitivity translates into excellent local control in extremity myxoid liposarcoma: a comparison with other soft tissue sarcomas. Cancer. 2009;115:3254-61.

5. Katz D, Boonsirikamchai P, Choi H, Lazar AJ, Wang WL, Xiao $\mathrm{L}$, et al. Efficacy of first-line doxorubicin and ifosfamide in myxoid liposarcoma. Clin Sarcoma Res. 2012;2:2.

6. Marshall S, Nakano K, Sugiura Y, Taira S, Ono M, Tomomatsu $\mathrm{J}$, et al. Outcome for advanced or metastatic soft tissue sarcoma of nonextremities treated with doxorubicin-based chemotherapy: a retrospective study from a single cancer institution. Sarcoma. 2018;2018:8926598.

7. National Comprehensive Cancer Network. Soft tissue sarcoma (version 6.2019) [Internet]. Plymouth Meeting, PA: National Comprehensive Cancer Network; 2020 [cited 2020 Feb 10]. Available from: https://www.nccn.org/professionals/ physician_gls/PDF/sarcoma.pdf.
8. Jones RL, Fisher C, Al-Muderis O, Judson IR. Differential sensitivity of liposarcoma subtypes to chemotherapy. Eur J Cancer. 2005;41:2853-60.

9. D'Angelo SP, Mahoney MR, Van Tine BA, Atkins J, Milhem MM, Jahagirdar BN, et al. Nivolumab with or without ipilimumab treatment for metastatic sarcoma (Alliance A091401): two open-label, non-comparative, randomised, phase 2 trials. Lancet Oncol. 2018;19:416-26.

10. Tawbi HA, Burgess M, Bolejack V, Van Tine BA, Schuetze SM, $\mathrm{Hu}$ J, et al. Pembrolizumab in advanced soft-tissue sarcoma and bone sarcoma (SARC028): a multicentre, two-cohort, single-arm, open-label, phase 2 trial. Lancet Oncol. 2017;18:1493501.

11. Powles T, O'Donnell PH, Massard C, Arkenau HT, Friedlander TW, Hoimes CJ, et al. Efficacy and safety of durvalumab in locally advanced or metastatic urothelial carcinoma: updated results from a phase 1/2 open-label study. JAMA Oncol. 2017;3:e172411.

12. Dalal KM, Antonescu CR, Singer S. Diagnosis and management of lipomatous tumors. J Surg Oncol. 2008;97:298-313.

13. Dalal KM, Kattan MW, Antonescu CR, Brennan MF, Singer S. Subtype specific prognostic nomogram for patients with primary liposarcoma of the retroperitoneum, extremity, or trunk. Ann Surg. 2006;244:381-91.

14. Knebel C, Lenze U, Pohlig F, Lenze F, Harrasser N, Suren $\mathrm{C}$, et al. Prognostic factors and outcome of liposarcoma patients: a retrospective evaluation over 15 years. BMC Can- 
cer. 2017;17:410.

15. Singer S, Antonescu CR, Riedel E, Brennan MF. Histologic subtype and margin of resection predict pattern of recurrence and survival for retroperitoneal liposarcoma. Ann Surg. 2003;238:358-70.

16. Anaya DA, Lahat G, Liu J, Xing Y, Cormier JN, Pisters PW, et al. Multifocality in retroperitoneal sarcoma: a prognostic factor critical to surgical decision-making. Ann Surg. 2009;249:137-42.

17. Kim C, Kim EK, Jung H, Chon HJ, Han JW, Shin KH, et al. Prognostic implications of PD-L1 expression in patients with soft tissue sarcoma. BMC Cancer. 2016;16:434.

18. D'Angelo SP, Shoushtari AN, Agaram NP, Kuk D, Qin LX, Carvajal RD, et al. Prevalence of tumor-infiltrating lymphocytes and PD-L1 expression in the soft tissue sarcoma microenvironment. Hum Pathol. 2015;46:357-65.

19. Kim JR, Moon YJ, Kwon KS, Bae JS, Wagle S, Kim KM, et al. Tumor infiltrating PD1-positive lymphocytes and the expression of PD-L1 predict poor prognosis of soft tissue sarcomas. PLoS One. 2013;8:e82870.

20. Paydas S, Bagir EK, Deveci MA, Gonlusen G. Clinical and prognostic significance of PD-1 and PD-L1 expression in sarcomas. Med Oncol. 2016;33:93.

21. Orth MF, Buecklein VL, Kampmann E, Subklewe M, Noessner E, Cidre-Aranaz F, et al. Expression patterns of PD-L1 and PD-1 provide rationales for immune checkpoint inhi- bition in soft tissue sarcomas. Preprint at http://doi.org/ 10.1101/569418 (2019).

22. Yan L, Wang Z, Cui C, Guan X, Dong B, Zhao M, et al. Comprehensive immune characterization and T-cell receptor repertoire heterogeneity of retroperitoneal liposarcoma. Cancer Sci. 2019;110:3038-48.

23. Zhu Z, Jin Z, Zhang M, Tang Y, Yang G, Yuan X, et al. Prognostic value of programmed death-ligand 1 in sarcoma: a metaanalysis. Oncotarget. 2017;8:59570-80.

24. Torabi A, Amaya CN, Wians FH Jr, Bryan BA. PD-1 and PDL1 expression in bone and soft tissue sarcomas. Pathology. 2017;49:506-13.

25. Tseng WW, Malu S, Zhang M, Chen J, Sim GC, Wei W, et al. Analysis of the intratumoral adaptive immune response in well differentiated and dedifferentiated retroperitoneal liposarcoma. Sarcoma. 2015;2015:547460

26. Keung EZ, Lazar AJ, Torres KE, Wang WL, Cormier JN, Ashleigh Guadagnolo B, et al. Phase II study of neoadjuvant checkpoint blockade in patients with surgically resectable undifferentiated pleomorphic sarcoma and dedifferentiated liposarcoma. BMC Cancer. 2018;18:913.

27. Keung EZ, Burgess M, Salazar R, Parra ER, Rodrigues-Canales J, Bolejack V, et al. Correlative analyses of the SARC028 trial reveal an association between sarcoma-associated immune infiltrate and response to pembrolizumab. Clin Cancer Res. 2020;26:1258-66. 